\title{
A rapid qualitative assay for detection of Clostridium perfringens in canned food products*
}

\author{
Gayatri Ashwinkumar Dave ${ }^{凶}$ \\ Department of Biotechnology, P D Patel Institute of Applied Sciences, Charotar University of Science and Technology (CHARUSAT), \\ Changa-388421, India
}

Clostridium perfringens (MTCC 1349) is a Gram-positive, anaerobic, endospore forming, and rod-shaped bacterium. This bacterium produces a variety of toxins under strict anaerobic environment. $C$. perfringens can grow at temperatures ranging between $20^{\circ} \mathrm{C}$ and $50^{\circ} \mathrm{C}$. It is the major causetive agent for gas gangrene, cellulitis, septicemia, necrotic enteritis and food poisoning, which are common toxin induced conditions noted in human and animals. C. perfringens can produce produce four major types of toxins that are used for the classification of strains, classified under type A-E. Across the globe many countries, including the United States, are affected by C. perfringens food poisonings where it is ranked as one of the most common causes of food borne infections. To date, no direct one step assay for the detection of C. perfringens has been developed and only few methods are known for accurate detection of $C$. perfringens. Long detection and incubation time is the major consideration of these reporter assays. The prensent study proposes a rapid and reliable colorimetric assay for the detection of C. perfringens. In principale, this assay detects the para nitrophenyl (yellow colour end product) liberated due to the hydrolysis of paranitrophenyl phosphetidyl choline (PNPC) through phospholipase C (lecithinase). Constitutive secretion of phospholipase $C$ is a charactristic feature of $C$. perfringens. This assay detects the presence of the extracellular lecithinse through the PNPC impragnated impregnated probe. The probe is impregnated with peranitrophenyl phosphotidyl choline ester, which is colourless substrate used by lecithinase. The designed assay is specific towards PNPC and detectes very small quantites of lecithinase under conditions used. The reaction is substrate specific, no cross reaction was observed upon incubation with other substrates. In addition, this assay gave negative results with other clostridium strains, no cross reactions were observed with other experimental strains like $C$. tetani, $C$. botulinum, $C$. acetobutyricum, Bacillus subtilis, and Escherichia coli. This assay is extramly rapid and provides reliable and reproducible results within one hour of incubation at $37^{\circ} \mathrm{C}$.

Key words: Clostridium perfringens; lecithinase; peranitrophenyl phosphotidyl choline ester

Received: 07 August, 2015; revised: 17 August, 2016; accepted: 09 April, 2017; available on-line: 09 June, 2017

*This paper was presented at the 6th International Weigl Conference on Microbiology and the abstract was published in Acta Biochemica Polonica.

e-mail: gayatridave.bt@charusat.ac.in

Abbreviations: $\mathrm{cm}$, centimetre; $h$, hour; RPM, revolutions per minute; CFU, colony forming unit; $\mathrm{nm}$, nanometre

\section{INTRODUCTION}

Canning is generally regarded as a safe method for preserving food over prolonged periods of time, if practiced properly. In the canning process, foodstuff kept in jars or similar containers is heated above the boiling temperature of water. This process kills the microbes without compromising the quality of the foodstuff. During this heating process, the air is driven out of the jar and as it cools a vacuum seal is formed. This vacuum seal prevents the air from getting back into the product and bringing with it possible contaminating microorganisms. Canning efficiently removes microbes. Occasionally, few anaerobic microbes and spores of anaerobes that survive under this harsh killing procedure may contaminate the food products.

Clostridium perfringens is a Gram-positive anaerobic spore-forming bacterium that causes life-threatening gas gangrene and mild enterotoxaemia in humans, although it colonizes humans and animals as a normal intestinal flora. The organism is known to produce a variety of toxins and enzymes that are responsible for the severe myonecrotic lesions. Among five types (A to $\mathrm{E}), C$. perfringens type $\mathrm{A}$ is known to cause two types of infectious diseases in humans. The first is gas gangrene (Clostridial myonecrosis), in which $C$. perfringens typically contaminates a skin wound as a spore from the soil and then rapidly grows and produces various histolytic toxins and enzymes that destroy the surrounding muscles and connective tissues. The second type of common disease is food poisoning caused through the few strains of Clostridia (approximately 5\% of all C. perfringens type A isolates) of $C$. perfringens producing enterotoxin (CPE) (Ohtani et al., 2013). The CPE-producing strains are responsible for nearly one million cases of $C$. perfringens food poisonings annually. It is also ranked as the second most commonly reported bacterial food-borne disease in the United States (Jihong Li et al., 2013). When food products contaminated with vegetative CPE-producing C. perfringens cells are ingested, the microbes colonize the intestine and begin to sporulate within 24-h of incubation. During the sporulation process, a large amount of CPE is produced. CPE forms pores in the membranes of intestinal epithelial cells and causes watery diarrhoea.

Presently, the reported bioassays detect clostridia through culturing on specific microbiological media. This culturing method requires a long time for detection and it also requires an extra effort to create oxygen free environment for growth. Countries in the developing world, however, require a rapid, inexpensive and simple method to perform tests that do not require a highly sophisticated laboratory and equipment. To address 
Table 1. Microbial cultures used for this study

\begin{tabular}{|c|c|c|c|}
\hline Name & Characteristics & Optimum growth conditions & Reference \\
\hline Clostridium perfringens (MTCC 450) & Lecithinase $^{+}$ & $\begin{array}{l}\text { Temperature } 30^{\circ} \mathrm{C} \text {, Reinforced Clostridial } \\
\text { Agar, In Anaerobic jar equipped with Ana- } \\
\text { ero-gas pack }\end{array}$ & This work \\
\hline Clostridium botulinum (ATCC 3502) & Botulin+, Lipase $^{+}$ & Temperature $37^{\circ} \mathrm{C}$, Clostridia agar & (Kirk et al., 2012) \\
\hline Clostridium perfringens (MTCC 450) & Lecithinase- & $\begin{array}{l}\text { Temperature } 30^{\circ} \mathrm{C} \text {, Fluid thioglycollate } \\
\text { broth }\end{array}$ & This work \\
\hline Saccharomyces cerevisiae YJF130 & PLC1 (phospholipase ${ }^{+}$ & $\begin{array}{l}\text { Temperature } 30^{\circ} \mathrm{C} \text {, Glucose yeast extract } \\
\text { broth }\end{array}$ & (Flick \& Thorner, 1993) \\
\hline E. coli DH5a & Wild type & Temperature $37^{\circ} \mathrm{C}$, Nutrient broth & - \\
\hline Bacillus subtilis & Wild type & Temperature $37^{\circ} \mathrm{C}$, Nutrient broth & - \\
\hline
\end{tabular}

the need of developing nations, this study presents a colorimetric paper-based one step assay for the detection of $C$. perfringens in canned food products (Fig. 1). The study further optimizes the assay parameters for rapid detection.

\section{MATERIALS AND METHODS}

Cultures, media and materials. This colorimetric biochemical reaction was studied with 6 bacterial species to evaluate the cross reactivity and the specificity of colour reaction. All of the cultures were obtained from Microbial Type Culture Collection Centre (MTCC) Chandigarh, INDIA and American Type Culture Collection Centre, Manassas, USA and are maintained in our laboratory. Culture conditions and their characteristics are listed in Table 1 . The cultures were bimonthly transferred into the cultivation medium and stored at $4{ }^{\circ} \mathrm{C}$ in the course of this study. All of the chemicals were purchased from Sigma-Aldrich. The key substrate, $\varrho$-nitro phenyl phosphetidyl choline, was purchased from SigmaAldrich as well.

Q-nitro phenyl phosphetidylcholine [PNPC] impregnated strip. Water and ethanol, mixed at an equal ratio, was used for preparation of 1\% PNPC solution for loading on an absorbent paper. Absorbent paper (Whatman No.1) was cut into $25 \mathrm{~cm}$ long strips, and the width of each strip was fixed at $1.5 \mathrm{~cm}$. Acetone $(60 \%)$ was used to remove water insoluble impurities attached to the absorbent paper; to remove them, the paper was immersed in the acetone solution and air dried for 2-h at room temperature. An end of the air-dried paper strip

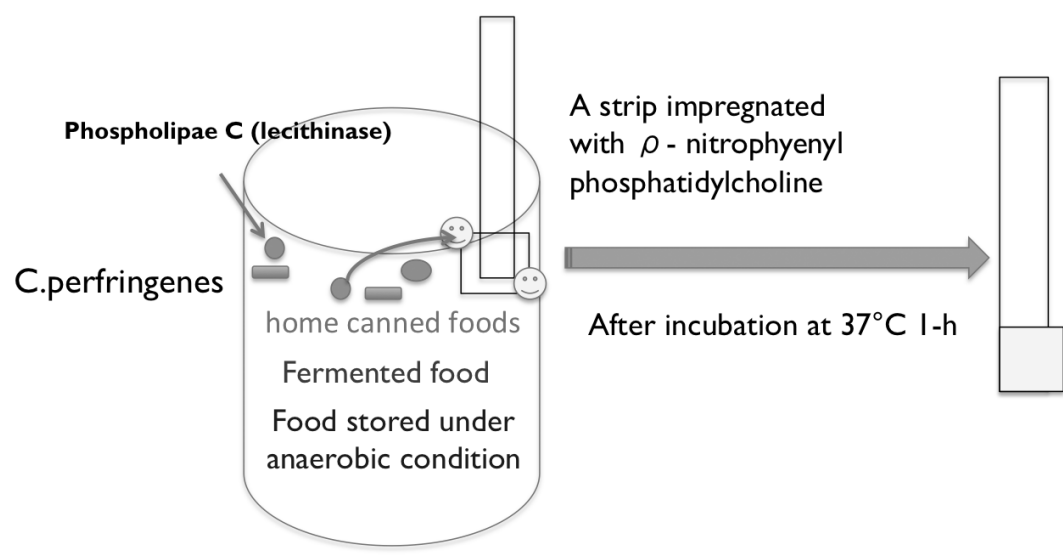

Figure 1. Principles of colorimetric assay
(10 cm long) was fixed to a wooden strip by an adhesive (purchased from a local market). This unfolded strip of paper was completely immersed in 800 microliters of the PNPC solution for nonspecific attachment of PNPC onto the paper surface. The strip was then dried in a hot air oven (EIE instruments) for 1-h and to remove traces of water the strip was further dried in a desiccator for 24-h. A completely dried strip was carefully rolled over the wooden support and stored in a sterile container for further application.

Substrate specificity of enzyme-substrate reaction; a quantitative estimation. An enzyme is a biomolecule that is very specific toward its substrate. In order to evaluate the substrate specificity of this developed biochemical assay, six different microbial cultures were incubated in 1\% PNPC and 1\% Para-nitrophenyl palmitate broth prepared in deionized distilled water. The initial density was adjusted at $100 \mathrm{CFU} / \mathrm{ml}$ for the six strains listed in Table 1 . This reaction mixture was incubated at $37^{\circ} \mathrm{C}$ for $1-\mathrm{h}$, followed by centrifugation (Sigma) at 4000 RPM for 10 minutes. Supernatants were collected from each reaction mix and the absorbance was measured at $450 \mathrm{~nm}$ in an UV-Visible spectrophotometer (Systronics). Purified lecithinase and lipase (Sigma-Aldrich) were used as standard controls.

Two Factor analysis; Microbial load and temperature. Canned foods are generally stored at a low temperature that ultimately slows down/prevents the growth of $C$. perfringens. Two-factor analysis was performed to evaluate the effect of temperature and microbial load on the designed assay. This analysis was carried out with different microbial load (measured by CFU analysis) in the range of $10^{1}-10^{11}$, at different series of temperatures $\left(2-40^{\circ} \mathrm{C}\right)$. To obtain the high-density bacterial biomass, two litters of cultivation broth containing pure bacterial cultures (O.D. $=0.8$ ) were subjected to centrifugation. Supernatants were discarded and the pellets were resuspended in phosphate buffered saline. The concentrated biomass was enumerated through Standard plate count, performed by a serial dilution method $\left(10^{8}\right)$ of the concentrated biomass. The stock culture for each concentration of microbial load was prepared by this technique. The cultures from the respective stock $\left(10^{1}-10^{11}\right)$ were then incubated at the reaction temperatures for optimization. The 
Table 2. Norm of reaction

\begin{tabular}{|c|c|c|c|c|}
\hline Microbial load & & Lecithinase (U/I) & $\stackrel{\circ}{\circ}$ & Color reaction \\
\hline 10 & 둗 & 0.002 & $\begin{array}{l}\text { 음 } \\
\text { 둠 }\end{array}$ & \\
\hline $10^{2}$ & $\underbrace{\grave{0}}_{\cup}$ & 32.3 & $\frac{\text { อ }}{\stackrel{0}{\nu}}$ & \\
\hline $10^{3}$ & $\stackrel{N}{m}$ & 42.6 & $\begin{array}{l}3 \\
\frac{3}{3} \\
0\end{array}$ & \\
\hline $10^{4}$ & ঐे & 48.9 & 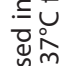 & \\
\hline $10^{5}$ & $\begin{array}{l}\text { 둥 } \\
\text { 응 }\end{array}$ & 44 & $\begin{array}{l}\frac{\omega}{d} \\
\varepsilon \\
\varepsilon \\
\varepsilon \\
\end{array}$ & \\
\hline $10^{6}$ & $\begin{array}{l}\bar{u} \\
\sum_{a}\end{array}$ & 45.8 & 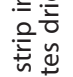 & \\
\hline $10^{7}$ &. & 52.5 & 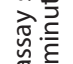 & \\
\hline $10^{8}$ & $\begin{array}{l}\stackrel{0}{\pi} \\
\frac{0}{2}\end{array}$ & 54.9 & ర్d & \\
\hline $10^{9}$ & $\underline{\varrho}$ & 69.9 & ○ & \\
\hline $10^{10}$ & & 92.1 & $\sum_{a}$ & \\
\hline
\end{tabular}

lecithinase activity was measured after 24-h incubation at different temperatures in the FTB broth.

Lecithinase assay: one millilitre of the culture supernatant was incubated in 1\% PNPC solution for 1-h; the liberated paranitrophenyl concentration was measured as an absorbance at $450 \mathrm{~nm}$.

Optimization of assay parameters. Reproducibility, cost and robustness are desirable features of any diagnostic assay. These parameters were optimized through the series of experiments. Experiments included measurement of critical substrate and enzyme concentration, and the efficiency of colour reaction in the presence of other microbes. The colour reaction was also assayed by incubating the microbes on heterogeneous substrates. The diagnostic range of the assay was determined by experiments that involved the very few clostridia (as few as $10 \mathrm{CFU} / \mathrm{ml}$ ) and increased to a very high number of Clostridia. Concurrently, in the same experiments, the lecithinase activity was also analysed for different microbial load. The colour intensity was recorded for each assay condition.

Validation of the mode of lecithinase action through FT-IR. IR spectra of solutions containing lecithin (a), PNPC (1\%) (b), PNPC (1\%) + lecithinase (1U) (c) were measured from $4000 \mathrm{~cm}^{-1}$ to $500 \mathrm{~cm}^{-1}$ with an FTIR spectrophotometer. An overhead-attenuated total refraction [ATR] accessory was equipped as the sample stage for liquid samples. All spectral measurements were done at $1 \mathrm{~cm}^{-1}$ resolution. The single beam ATR spectrum from each sample was corrected using a background spectrum of deionized water, and transformed to absorbance units.

\section{RESULTS AND DISCUSSIONS}

\section{Colour reaction and its specificity assessment}

This method of detection of $C$. perfringens is very easy to perform and offers an excellent solution for developing nations. In the presence of $C$. perfringens, the paper strips impregnated with PNPC turn yellow (T). This reaction is very quick, as it gives the positive results just within $1-\mathrm{h}$ of incubation at $37^{\circ} \mathrm{C}$. The developed colour reaction $(T)$ is stable, no change was observed even after 24-h observation (Fig. 2), while the control strip has not shown any colour change in the absence of $C$. perfringens.

A pre-validation test (Table 2) was performed to estimate the assay performance at various microbial loads, as sometimes the canned food products contain only a very few bacteria and are stored at ultralow temperatures that do not allow for the growth of microorganisms, hence an ideal assay should detect a very small number of microbes. Apparently, less numbers of $C$. perfringens have secreted little quantity of alpha toxin, insufficient for bringing the colorimetric change.

Our experiments show that even at the very low concentration of alpha-toxin (lecithinase), such as 0.002 $(\mathrm{U} / \mathrm{l})$, the developed strip shows a slight colour change, however, a better result was obtained when C. perfringens numbers exceeded 100 . The assay procedure presented in Table 2 was repeated 20 times in different laboratory

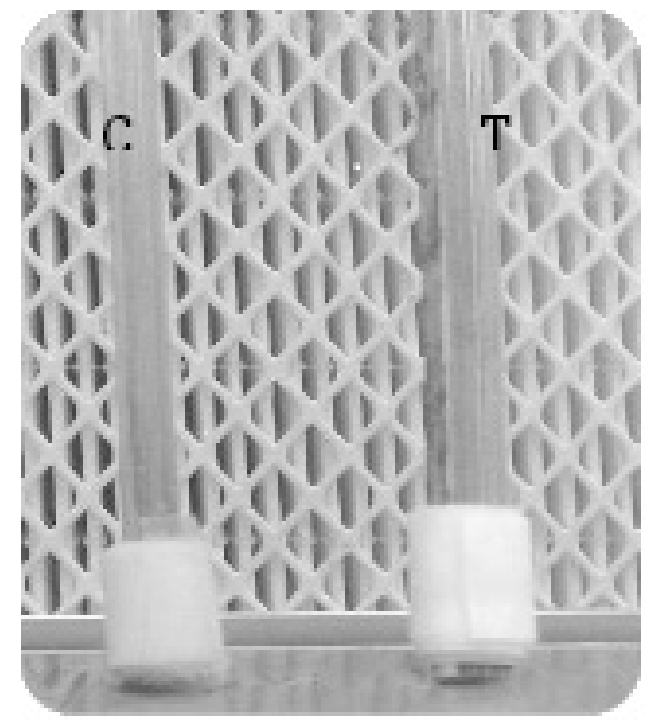

Figure 2. Control flask (C) contained FTB broth inoculated with an E. coli culture (100 CFU/ml).

Nonappearance of colour reaction in control strips indicates the unambiguous reaction of PNPC with lecithinase and its specificity towards C. perfringens. 


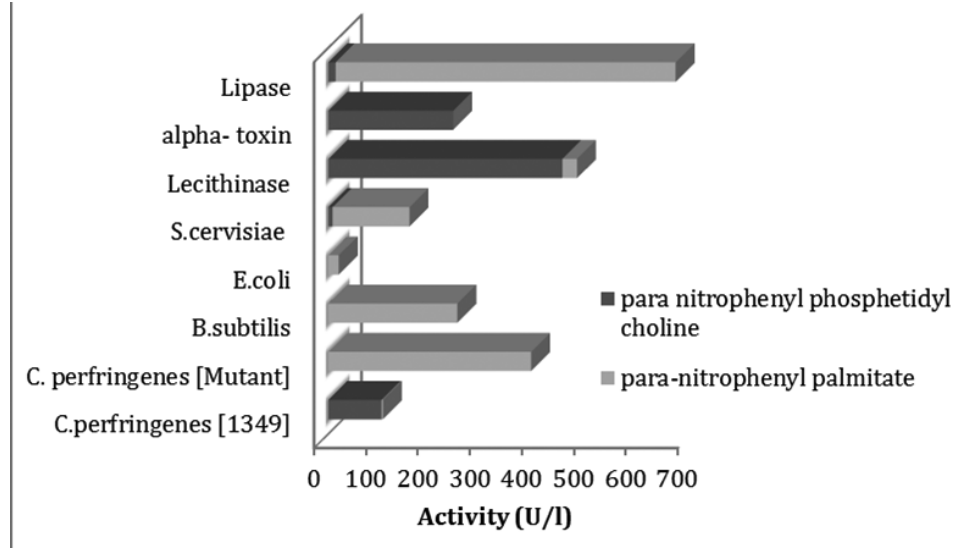

Figure 3. Estimation of lecithinase and lipase activity in different microbial species

and field environments to ensure the accuracy of the assay. The mean values of alpha toxins (lecithinase) are presented in Table 2 as well.

This assay is unequivocally sensitive for targeted pathogenic microbes. It is extremely fast when compared to other, previously reported assays for $C$. perfringens. These assays are either PCR based (which takes at least 4-h for preparation and detection) or are immunoassays which takes longer incubation time (Kanakaraj et al., 1998; Kokai-kun et al., 1994; Amar et al., 2002). In reported assay, the colour development was observed within 30 -minutes of incubation when the concentration of alpha-toxin was high. However, in the presence of low concentration of the alpha-toxin, it takes longer for colour development to take place (1-h), thus the optimized time for this assay is $1-\mathrm{h}$. In addition, the temperature does not exert any significant effect on the colour development reaction, i.e. an increase or decrease by $10^{\circ} \mathrm{C}$ seems to be insignificant (not shown).

Any procedure is meaningful when it is specific for intended use only. The strip is impregnated with $\varrho$-nitro phenyl phosphetidylcholine, which is a substrate for phospholipase C. PNPC is a natural phospholipid that may be utilized as a substrate by other nonspecific lipases. To evaluate the specificity of this designed substrate only for phospholipase $\mathrm{C}$, the biochemical reaction was evaluated with different microbial species that produced other classes of lipases. Purified lipase, lecithinase (plant source) and alpha toxin (lecithinase from C. perfringens) were also used as a control. The results presented in Fig. 3 confirm the specificity of the colour reaction for the designed assay. No significant lipase activity was observed in the reaction vials containing $C$. perfringens in 1\% PNPC broth and for $C$. perfringens in para nitrophenyl palmitate broth. In contrast, the lecithinase mutant strain of $C$. perfringens has shown a significant lipase activity in $1 \%$ para nitrophenyl palmitate broth (PNPB) but no activity in the PNPC broth. Lipolytic enzymes of C. perfringens have shown high substrate specificity towards their respective substrates. These results narrow down the chance of false detection, as no activity was detected for any other microbial genus studied in the present study. There are reports in the literature that the secretion of extracellular phospholipase $\mathrm{C}$ occurs in many other microbial species. Such reported species are Pseudomonas (Sacherer et al., 1994), Acinetobacter (Vallenet et al., 2008), and Bacillus anthracis (Read et al., 2003), and they can utilize PNPC through phospholipase C secretion. These species might constitute a threat towards the specificity of this assay for C. perfringens, but the complete absence of oxygen in canned food products prevents the growth of the mentioned aerobes. Thus, the canned food product preparation procedure in fact increases the accuracy of the designed assay towards the C. perfringens detection. In our analysis, this assay procedure has shown $100 \%$ accuracy in developing colour reaction with C. perfringens only. However, improper storage of food can allow the growth of other aerobes that might interfere with the detection through production of various metabolites. Hence, the chances of false positive or false negative results cannot be completely eliminated. The result of this assay should be confirmed with another microbiological medium based assay.

\section{Effect of storage temperature and microbial load on the enzyme activity; a two-component analysis}

Canned food products are generally stored at very low temperature, and this adversity may affect the extracellular lecithinase and make it difficult to detect by the current assay. To check this hypothesis, different numbers of $C$. perfringens were incubated for $24-h$ at different temperatures in tightly closed screw cap vials kept in the anaerobic chamber. The results show that with increasing storage temperature, the enzyme activity increases up to 100 folds (Fig. 4).

Though temperature significantly affects the lecithinase stability, a higher number of $C$. perfringens cells gave a positive reaction; a detectable enzyme activity was recorded even nearing the freezing temperature of $2^{\circ} \mathrm{C}$. The colorimetric detection method is very sensitive as it gives positive colour reaction at very low concentration of microbes, i.e. as little as 100 bacteria. In principle, this assay gives a positive colour reaction by the enzyme-mediated catalysis of PNPC, hence it is extremely important to determine the critical concentration of the enzyme for reaction to occur. This reaction can be obtained at very low concentration of enzyme, $0.002 \mathrm{U} / 1$ activity of lecithinase is sufficient for the colorimetric detection. At this concentration the colour is very faint yellow, and a minimum $1000 \mathrm{CFU}$ of C. perfringens are considered desirable for detectable colour development. This experiment also demonstrates the significant effect of the stor-

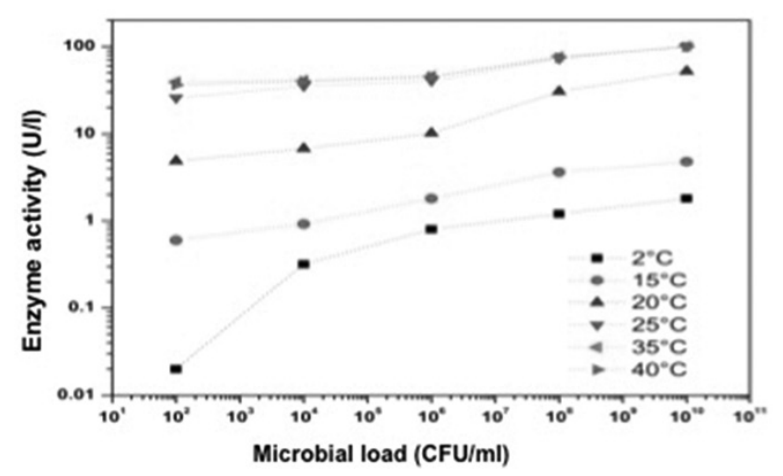

Figure 4. Effect of the storage temperature and the number of C. perfringens on the enzyme activity 
Table 3. Study of the efficiency of colour reaction in the presence of different substrate and Clostridia species

\begin{tabular}{|c|c|c|c|c|c|}
\hline & C.perfringens & C. botulinum & C. tetani & C. difficile & C. sordellii \\
\hline $2 \%$ starch & Yes & No & No & No & No \\
\hline $2 \%$ peptone & Yes & No & No & No & No \\
\hline $2 \%$ egg yolk & Yes & Variable* & No & No & No \\
\hline $2 \%$ olive oil & Yes & No & No & No & No \\
\hline Nutrient broth & Yes & No & No & No & No \\
\hline FTB & Yes & Variable* & Variable $e^{* *}$ & No & No \\
\hline Mineral Medium & No & No & No & No & No \\
\hline
\end{tabular}

*The positive reaction was observed for the culture growing below $20^{\circ} \mathrm{C}$. ${ }^{*}$ This culture gave positive reaction only in the presence of thioglycollate

age temperature and microbial load that are critical factors for detection.

\section{Effect of heterogeneous substrate on color reaction}

The genus Clostridia is extremely diverse in nature. It is closely related to many species, which are phylogenetically intermixed with other spore forming and non-spore forming genera that include more than 35 different species (Collins et al., 1994). Most likely, the other clostridium species can give a colour reaction with PNPC too; to investigate the risks of this cross-species reaction, the following experiment was performed. Five different Clostridia species were inoculated in media containing different substrates. A variety of canned food products is available on the market and contains a variety of macromolecules. C. perfringens can utilize other substrates by producing another set of enzymes. To evaluate the substrate-dependency of alpha-toxin production, an assay was performed with a variety of substrates. The alpha-toxin production was evaluated in the presence of starch, protein and lipid as a sole substrate and the results of this independent evaluation are summarized in Table 3.

Five Clostridia species were incubated with various substrates at 4 different temperatures i.e. $2^{\circ} \mathrm{C}, 20^{\circ} \mathrm{C}$, $37^{\circ} \mathrm{C}, 40^{\circ} \mathrm{C}$. This table summarizes the results for the whole temperature range, as no effect of temperature was observed for all cases tested, except three instances noted in the Table 3

The results presented in Table 3 further corroborate the specificity of our color reaction assay. It also sheds light on the pattern of alpha toxin production in C. perfringens. C. perfringens constitutively produce alpha toxin independent of the nature of substrate and temperature. Our results demonstrate for the first time the temperature dependent alpha toxin production in C.botulinum, however, this result does not pose any major threat towards the specificity of our assay as most of the canned food products are stored below $20^{\circ} \mathrm{C}$. The results obtained in the FTB medium may pose a constraint toward the species specific detection through this assay as the selected strain of $C$. botulinum and $C$. tetani both gave positive color reaction when grown in FTB. In order to study this constraint in detail, the knockout FTB media were prepared for each individual component. Striking- ly, in the absence of thioglycollate both of the species gave a negative color reaction. This primary study is not sufficient to establish any relationship between the presence of thioglycollate and alpha-toxin production, however, this study leaves an open-ended question for further research. The review of Hatheway (1990) suggests some interesting factors that are common for more than one species or group. Such common metabolites that increase the pathogenicity of microorganisms are neurotoxins, lethal toxins, lecithinases, oxygen-labile hemolysins, binary toxins, and ADP-ribosyltransferases. This review suggests that the Phospholipase $C$ (lecithinase) production is restricted to only the $2-3$ species. The $\mathrm{Ha}$ theway and other researchers' results indirectly support the specificity of the assay that we have developed.

\section{Effect of microbial consortia on the color reaction}

The presence of other bacteria might affect the color reaction. To evaluate the influence of other bacterial species on our assay, a combinatorial approach was used. Two different cultures were incubated in nutrient glucose broth for $72-\mathrm{h}$ at $37^{\circ} \mathrm{C}$ and were tested in through the design our colorimetric assay. The results are summarized in Table 4.

This study represents the different combinations of bacterial species, mixture of aerobes and anaerobes, mixture of aerobes and facultative anaerobes, mixture of anaerobes and facultative aerobes.

Interestingly, the combination of anaerobes and yeast results in the growth of $C$. perfringens even under anaerobic conditions, which may be the result of

Table 4. Influence of other microbes and growth conditions on the colour reaction assay

\begin{tabular}{lll}
\hline Condition & Complex & Reaction \\
\hline Aerobic & C. perfringens + S. cerevisiae & Yes \\
\hline & C. perfringens + E. coli & No \\
\hline & C. perfringens + Clostridium species & Yes \\
\hline Anaerobic & C. perfringens + S. cerevisiae & Yes \\
& C. perfringens + E. coli & Yes \\
\hline C. perfringens + Clostridium species & Yes \\
\hline
\end{tabular}




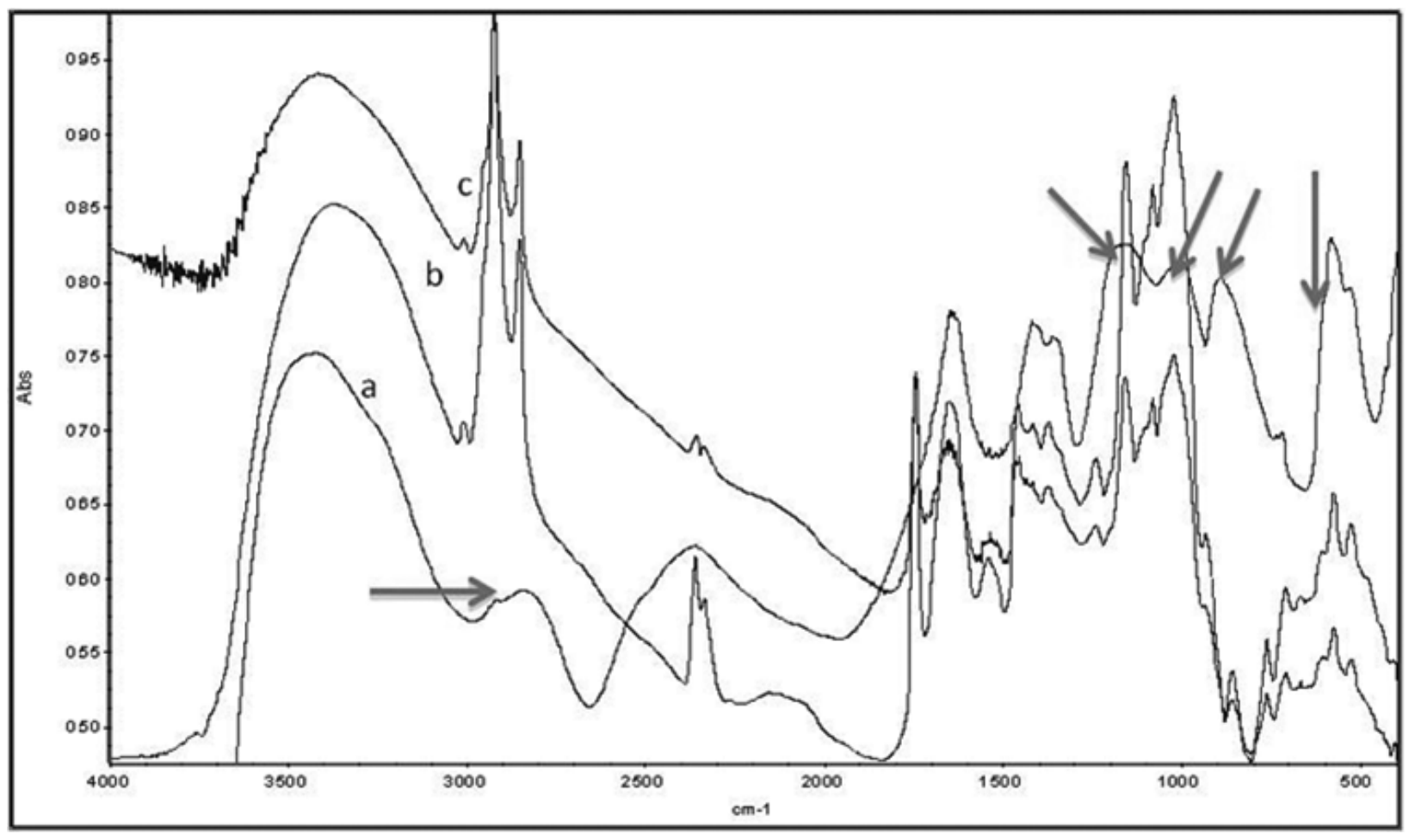

Figure 5. FT-IR spectra of:

(a) PNPC+lecithinase. (b) PNPC (c) PNPC+lipase single arrow indicates the peak of para-nitrophenyl which is absent in PNPC+lecithinase

the fermentation byproduct (carbon dioxide) produced by the $S$. cerevisiae yeast. It suggests that carbon dioxide produced by yeast results in creating the anaerobic environment for the growth of Clostridia. This result further confirms that this color reaction is not influenced by the presence of other microbes. A combination comprising the E. coli and C. perfringens has given a negative result in the aerobic environment as predicted, because $C$. perfringens fails to grow in the presence of atmospheric oxygen.

\section{FT-IR analysis for validation of the proposed mechanism}

The fact that phospholipase C can hydrolyze phosphatidylcholine was reported by many research groups (Sheikhnejad \& Srivastava, 1986; Itabe et al., 1994) and the fact that para-nitrophenyl butyrate can serve as a substrate for lipase is broadly reported by many researchers. Itabe et al. (1994) has reported the interaction of PNPC with lecithinase and that has greatly inspired the work presented here. The action of lecithinase liberates the para-nitrophenyl from PNPC and this was reported even earlier in the $20^{\text {th }}$ century (Thorpe \& Miller, 1981). These findings were neglected for a very long period of time and the reported colour reaction was never utilized for the development of a colorimetric assay. To re-confirm these two-decade-old findings, FT-IR analysis was performed (Fig. 5).

Rodriguesand coworkers (2007) has reported FT-IR spectrum of para-nitrophenyl phosphetidyl choline and phosphatidylcholine. The para-nitrophenyl group gave specific peak at the wave number $3000 \mathrm{~cm}^{-1}$ that was found to be completely missing in the sample which was incubated with PNPC lecithinase. The similar peak of para-nitrophenyl was seen in PNPC+ lipase that indicates that the lipase cannot liberate para-nitrophenyl group from phosphatidylcholine. Pure lecithinase can remove the para-nitrophenyl group from phosphetidylchholine. The FT-IR spectrum suggests the lipase is inducing some change in the functional group of PNPC (multiple blue arrows) but it does not catalyze the hydrolysis of para-nitrophenyl. It further commemorates the specificity of the developed assay.

In summary, our colorimetric assay is reliable and species-specific when performed properly. This assay is designed and optimized for canned food products. The temperature, presence of other bacteria and the nature of food products do not hinder the development of the colour reaction.

\section{CONCLUSION}

This assay can be employed for all: the solid, liquid and semi-solid food products with little modification in the assay procedure. This study concludes with two open ends for research; one is the effect of thioglycollate on production of the alpha toxin and the second is the effect of temperature on the alpha toxin production by C.botulinum. This simple assay can be employed anywhere and by anyone, and the only requirement for development of the colour reaction is the ability to maintain a proper temperature. In a country like India, the atmospheric temperature is always above $30^{\circ} \mathrm{C}$, except for the winter and during the night. As mentioned above, at temperatures over $30^{\circ} \mathrm{C}$, the production of alpha toxin by $C$. botulinum may occur - this problem needs further investigation. The assay we have developed gave colour reaction at room temperature in India. The research we presented here may open the door for the routine screening of canned food products for C. perfringens. 


\section{REFERENCES}

Berry PR, Rodhouse JC, HughesS, Bartholomew BA, Gilbert RJ (1988) Evaluation of ELISA, RPLA, and Vero cell assays for detecting Clostridium perfringens enterotoxin in faecal specimens. I Clin Pathol 41: 458-461. PMCID: PMC1141476

Collins MD, Lawson PA, Willems A, Cordoba JJ, Fernandez-Garayzabal J, Garcia P, Cai J, Hippe H, Farrow JA (1994) The phylogeny of the genus Clostridium: proposal of five new genera and eleven new species combinations. Int J Syst Bacteriol 44: 812-826. DOI: 10.1099/00207713-44-4-812

Flick JS, Thorner J (1993). Genetic and biochemical characterization of a phosphatidylinositol-specific phospholipase C in Saccharomyces cerevisiae. Mol Cell Biol 13: 5861-5876

Hatheway CL (1990). Toxigenic clostridia. Clin Microbiol Rev 3: 66

Itabe $\mathrm{H}$, Takeshima E, Iwasaki H, Kimura J, Yoshida Y, Imanaka T, \& Takano T (1994). A monoclonal antibody against oxidized lipoprotein recognizes foam cells in atherosclerotic lesions. Complex formation of oxidized phosphatidylcholines and polypeptides. J Biol Chem 269: 15274-15279

Kirk DG, Dahlsten E, Zhang Z, Korkeala H, Lindström M (2012) Involvement of Clostridium botulinum ATCC 3502 sigma factor $\mathrm{K}$ in early-stage sporulation. Appl Environ Microbiol 78: 4590-4596. doi:10.1128/AEM.00304-12

Jihong L, Adams V, Bannam TL, Miyamoto K, Garcia JP, Uzal FA, Rood JI, McClane BA (2013) Toxin plasmids of Clostridium perfringens. Microbiol Mol Biol Rev 77: 208-233. doi: 10.1128/ MMBR.00062-12
Read TD, Peterson SN, Tourasse N, Baillie LW, Paulsen IT, Nelson KE, Hanna PC (2003) The genome sequence of Bacillus anthracis Ames and comparison to closely related bacteria. Nature 423: 81-86: doi: $10.1038 /$ nature 01590

Kanakaraj R, Harris DL, Songer JG, Bosworth B (1998). Multiplex PCR assay for detection of Clostridium perfringens in feces and intestinal contents of pigs and in swine feed. Vet Microbiol 63: 29-38. https://doi.org/10.1016/S0378-1135(98)00229-6

Kokai-Kun JF, Songer JG, Czeczulin JR, Chen F, McClane BA (1994) Comparison of Western immunoblots and gene detection assays for identification of potentially enterotoxigenic isolates of Clostridium perfringens. I Clin Microbiol 32: 2533-2539

Rodrigues AS, Aguiar APD, de Aguiar MR, Santa Maria LCD (2007) Quaternization reaction of 2-vinylpyridine and 4-vinylpyridine network copolymers for 4-nitrophenol adsorption. J Brazil Chem Soc 18: 431-436. http://dx.doi.org/10.1590/S0103-50532007000200028

Sheikhnejad RG, Srivastava PN (1986) Isolation and properties of a phosphatidylcholine-specific phospholipase C from bull seminal plasma. I Biol Chem 261: 7544-7549

Soutourina OA, Monot M, Boudry P, Saujet L, Pichon C, Sismeiro O, Semenova E, Severinov K, Le Bouguenec C, Coppée JY, Dupuy B (2013) Genome-wide identification of regulatory RNAs in the human pathogen Clostridium difficile. PLoS Genet 9: e1003493. https:// doi.org/10.1371/journal.pgen.1003493

Thorpe TC, Miller RD (1981). Extracellular enzymes of Legionella pneumophila. Infection and Immunity 33: 632-635 